\title{
A Novel OFDM Chirp Waveform Scheme for Use of Multiple Transmitters in SAR
}

\author{
Jung-Hyo Kim, Member, IEEE, Marwan Younis, Senior Member, IEEE, \\ Alberto Moreira, Fellow, IEEE, and Werner Wiesbeck, Fellow, IEEE
}

\begin{abstract}
In this letter, we present a new waveform technique for the use of multiple transmitters in synthetic aperture radar (SAR) data acquisition. This approach is based on the principle of the orthogonal-frequency-division-multiplexing technique. Unlike multiple subband approaches, the proposed scheme allows the generation of multiple orthogonal waveforms on common spectral support and thereby enables to exploit the full bandwidth for each waveform. This letter introduces the modulation and the demodulation processing in regard to typical spaceborne SAR receive signals. The proposed processing techniques are verified by a simulation for the case of pointlike targets.
\end{abstract}

Index Terms-Digital beamforming (DBF), multiple-input multiple-output (MIMO) synthetic aperture radar (SAR), orthogonal frequency division multiplexing (OFDM), orthogonal waveform, SAR.

\section{INTRODUCTION}

$\mathbf{T}$ HE USE of multiple transmitters is of a great interest in the synthetic aperture radar (SAR) community. It provides an increase of degrees of freedom and dramatically improves SAR imaging performance when combined with multiple receivers, a so-called multiple-input multiple-output (MIMO) constellation [1]. However, the waveform design for multiple transmitters has been the most important and challenging issue in realizing the MIMO SAR concept. Particularly for spaceborne SAR, aside from the orthogonality between waveforms for simultaneous multiple pulse transmissions, a constant envelope of the waveform is desired, considering the high power amplifier (HPA) in transmitters. The HPAs in spaceborne SAR systems operate in saturation in order to generate the maximum output power and to ensure a stable output power level for amplitude variations in the HPA input signal [2]. A significant envelope variation of waveform therefore yields a clipping effect on the output waveform, and the orthogonality can be broken.

This letter presents a novel waveform scheme to meet the aforementioned requirements, based on the principle of orthogonal frequency division multiplexing (OFDM) proposed in [3]. In OFDM systems, an available signal bandwidth is divided into multiple subbands, which is specified to be narrower than the

Manuscript received April 20, 2012; revised June 29, 2012; accepted July 16,2012

J.-H. Kim, M. Younis, and A. Moreira are with the Microwaves and Radar Institute, German Aerospace Center (DLR), 82234 Oberpfaffenhofen, Germany (e-mail: junghyo.kim@dlr.de).

W. Wiesbeck is with the Institute of High Frequency Technique and Electronics, Karlsruhe Institute of Technology, 76131 Karlsruhe, Germany.

Color versions of one or more of the figures in this paper are available online at http://ieeexplore.iee.org.

Digital Object Identifier 10.1109/LGRS.2012.2213577 channel coherence bandwidth, in order to avoid the frequencyselective channel effect [4]. The OFDM signaling is also paid attention for SAR applications, and recently, SAR processing techniques for OFDM waveforms were introduced in [5] and [6]. A major problem of the typical OFDM signal (mainly in spaceborne SAR) is the fast variation of the signal envelope. We resolve the problem by combining the OFDM principle with chirp waveforms. Therefore, the basic idea behind the proposed waveform scheme is to exploit both the orthogonality of subcarriers and intrinsic characteristics of traditional chirp waveforms.

The importance of using chirp waveform in the proposed scheme is emphasized in the following issues: First, one can easily achieve the constant envelope of time domain waveforms aforementioned, which leads to a maximum efficiency of the transmitter modules of a phased-array antenna. Second, the chirp spectrum approaches a rectangular shape as the time-bandwidth product increases so that its spectral efficiency and signal-to-noise ratio can be maximized [2], [7]. Finally, one can further exploit the linear frequency-time characteristics of chirp in signal processing. It makes it easy to combine the proposed scheme with existing SAR processing algorithms, such as the dechirp-on-receive technique.

Focusing on a dual transmit antenna scenario, we develop the novel waveform scheme consisting of the modulation and demodulation algorithm, based on the conventional OFDM processing, and validate its potential. Special attention has to be paid to the demodulation since the classical OFDM demodulation assumes that the delay length of a received signal is shorter than the length of the cyclic prefix (CP), but a SAR echo signal is generally much longer than the transmitted pulse length. In this letter, we present a demodulation strategy for such a long SAR signal.

\section{OFDM CHIRP MODULATION}

\section{A. Principles}

The basic idea behind the proposed technique is to exploit the orthogonality of discrete frequency components, i.e., subcarriers. This means that the orthogonality of waveforms is independent of the types of input sequences.

Assume the input sequence (spectrum) $\mathbf{S}[\bar{p}]$ with $N$ discrete spectral components, which are separated by $2 \Delta f$ as shown in Fig. 1. First, the input sequence $\mathbf{S}[\bar{p}]$ is interleaved by $N$ zeros, which is $\mathbf{S}_{1}[p]$ in Fig. 1, and then, the interleaved input sequence is shifted by $\Delta f$ for the second data sequence $\mathbf{S}_{2}[p]$. These data sequences are transformed into the time domain by the $2 N$-point inverse discrete Fourier transform (IDFT), which 


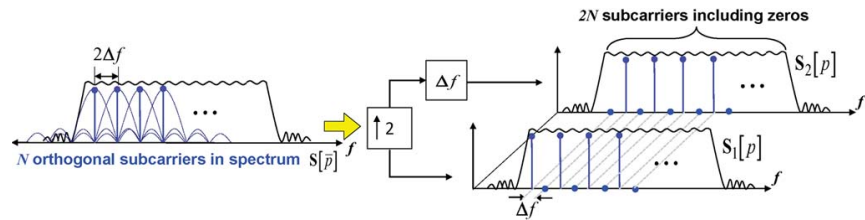

Fig. 1. Two orthogonal OFDM chirp waveforms are generated by zero interleaving and shift of a single chirp spectrum as an input sequence.

is the OFDM modulation. As a result, we obtain two waveforms modulated by two orthogonal subcarrier sets that are mutually shifted by $\Delta f$. Thus, their demodulation must be performed by $2 N$-point DFT. It must be emphasized that both the sets contain $2 N$ subcarriers but use only $N$ subcarriers to carry the input data, respectively.

\section{B. Signal Model}

As aforementioned, a chirp signal spectrum is used for the OFDM modulation in this work. The chirp signal spectrum, as input complex data, is obtained by

$$
S[\bar{p}]=\mathcal{F}\{s[n]\}=\mathcal{F}\left\{\exp \left(j \cdot \pi \cdot K_{r} \cdot\left(n T_{s}\right)^{2}\right)\right\}
$$

where $s[n]$ denotes the discrete time samples of a complex chirp signal with the length of $N, \mathcal{F}\{\cdot\}$ is the Fourier transform operator, $T_{s}$ is the sampling interval, and $K_{r}$ is the chirp rate, which is a ratio between the signal bandwidth $B$ and the chirp duration $T_{p}\left(K_{r}=B / T_{p}\right)$. Using (1), we generate two input data sequences by the zero interleaving and shift as follows:

$$
\begin{array}{lll}
\mathbf{S}_{1}[p]=[S[0], 0, S[1], 0, & \cdots & , S[N-1], 0] \\
\mathbf{S}_{2}[p]=[0, S[0], 0, S[1], & \cdots & , 0, S[N-1]]
\end{array}
$$

where $p=0,1,2, \ldots, 2 N-1$. Both data sequences contain total $2 N$ components, respectively. Therefore, they are modulated by $2 N$-point IDFT. According to the Cooley-Tukey algorithm, the DFT/IDFT can be performed by separate transforms with respect to the odd and even components of the input. Since the even components in $\mathbf{S}_{1}$ are zeros, the inverse Fourier transform of $S_{1}[p]$ is equivalent to that of $S[\bar{p}]$. Substituting $\bar{p}$ for $p / 2$, the modulated waveform $s_{1}$ is given by

$$
s_{1}[n]=\sum_{\bar{p}=0}^{N-1} S[\bar{p}] \cdot \exp \left(j \frac{2 \pi}{N} \bar{p} n\right)
$$

where $n=0,1,2, \ldots, 2 N-1$. Since the inverse Fourier transform of $S[\bar{p}]$ is $s[n]$ with the period of $N, s_{1}[n]$ can be expressed by a repetition of $s[n]$ over the length of $2 N$

$$
s_{1}[n]=s[n] \cdot \operatorname{rect}\left[\frac{n}{N}\right]+s[n-N] \cdot \operatorname{rect}\left[\frac{n-N}{N}\right] .
$$

In the same way, using $(p+1) / 2=\bar{p}+(1 / 2)$, the other modulated waveform $s_{2}$ is derived as

$$
s_{2}[n]=\exp \left(j \frac{\pi}{N} n\right) \sum_{\bar{p}=0}^{N-1} S[\bar{p}] \cdot \exp \left(j \frac{2 \pi}{N} \bar{p} n\right)
$$

and using $s[n]$, it can be also described as

$$
\begin{aligned}
s_{2}[n]= & \left(s[n] \cdot \operatorname{rect}\left[\frac{n}{N}\right]+s[n-N] \cdot \operatorname{rect}\left[\frac{n-N}{N}\right]\right) \\
& \cdot \exp \left(j \frac{\pi}{N} n\right) \\
= & s_{1}[n] \cdot \exp \left(j \frac{\pi}{N} n\right) .
\end{aligned}
$$

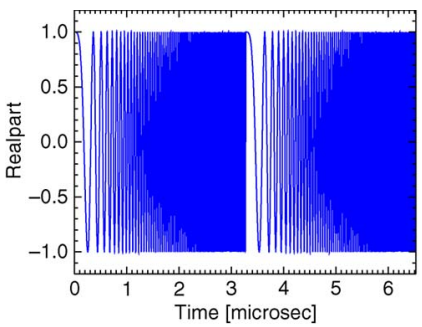

(a)

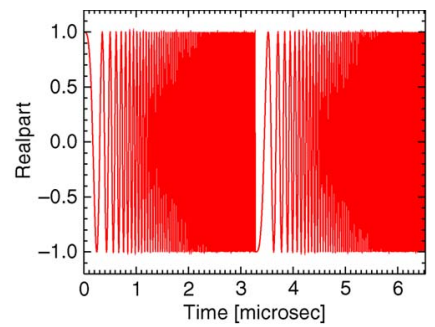

(b)
Fig. 2. Real parts of OFDM chirp waveforms in time domain with 50-MHz bandwidth and $N=4096$. The phase change in $s_{2}(t)$ due to the subcarrier offset must be remarked in comparison with $s_{1}(t)$. (a) $s_{1}(t)$. (b) $s_{2}(t)$.

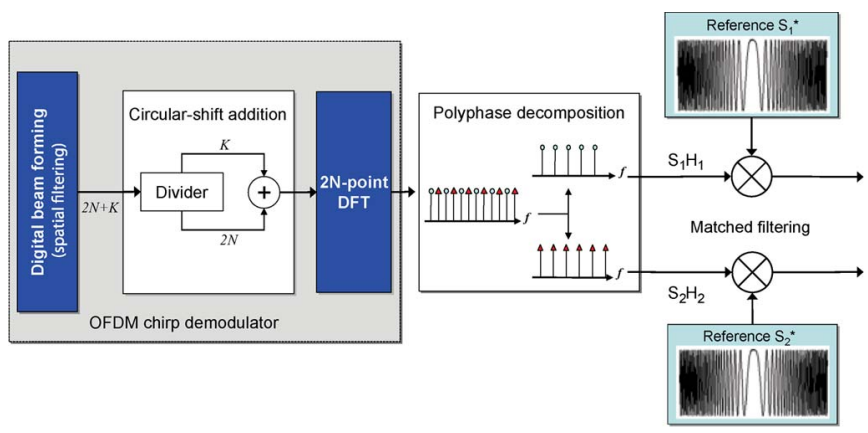

Fig. 3. Generic schematic of the proposed OFDM demodulator followed by the polyphase decomposition and parallel matched filters for the range compression.

By introducing $t=n T_{s}$ and $\Delta f=1 / 2 N T_{s}$, it is noticed that both waveforms are distinguishable by the subcarrier offset $\Delta f$. These modulated OFDM waveforms are converted to analog forms by a digital-to-analog converter. The OFDM waveforms in the time domain are plotted in Fig. 2. Due to the zero interleaving in the spectrum, the peak power level of the waveforms will be reduced. However, according to Parseval's theorem [8], the total energy of the input sequence is conserved.

Using the aforementioned signal model, the OFDM waveforms can be also directly produced in the time domain. The time domain generation is valuable since a band-limited input sequence can cause an overshoot in time domain waveforms [8].

\section{OFDM SAR SIGNAL DEMODULATION}

This section is dedicated to the development of an OFDM demodulation algorithm applicable to a received SAR signal, which is typically much longer than the OFDM pulse length. According to the classical OFDM demodulation scheme, the orthogonality of subcarriers can be exploited only if the following requirements are strictly fulfilled: synchronization between the modulator and demodulator in length and no truncation of the waveforms within IDFT/DFT windows. To meet the requirements, the demodulation algorithm developed in this section consists of the segmentation of the echo signal by spatial filtering (digital beamforming), the circular-shift addition, and the conventional OFDM demodulation using DFT, as shown in Fig. 3.

First of all, the spatial filters divide the echo signal into multiple subsets satisfying Lemma 1 , which will be derived in the next section. Second, the circular-shift addition is carried out so that $2 N$ samples from each spatial filter are obtained. As 


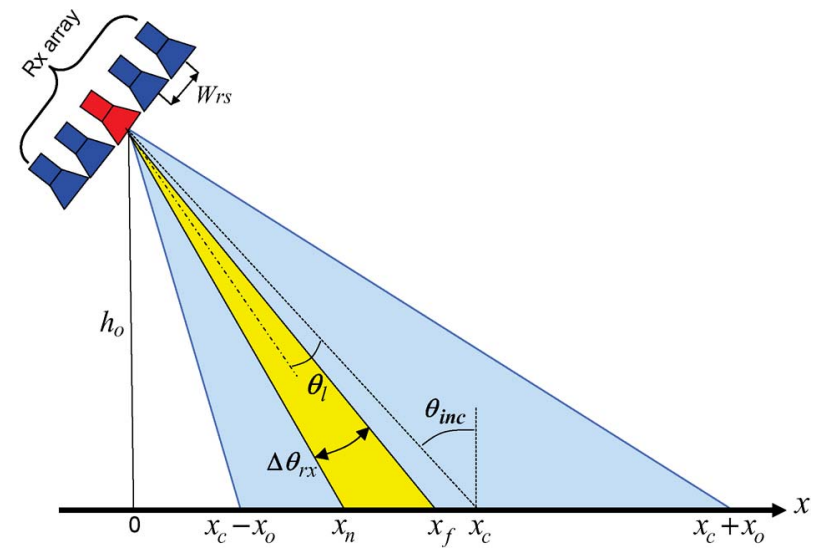

Fig. 4. Spatial filter pattern parameters and geometric parameters. The wide pattern indicates the transmit antenna beam illuminating the whole swath, and the narrow pattern depicts the main lobe of a spatial filter beam.

a conventional OFDM demodulation, the $2 N$-point DFT block demodulates the OFDM signals. Consequently, the polyphase decomposition block easily separates the waveforms. The decomposed signals are independently compressed by parallel matched filters.

\section{A. Segmentation: Spatial Filtering [Digital Beamforming $(D B F)]$}

A careful choice of weighting factors allows the steering of a sharp and high-gain beam to the corresponding angular region. This angular sectoring is called spatial filtering, which is an important functionality of digital beamforming. This process divides a whole swath into multiple subswaths so that the echo length of each subswath is significantly reduced. The spatial filter is time invariant, providing a constant gain over time, and its pattern only varies with the elevation angle.

A signal model for the spatial filtering is developed on the flat Earth model. Fig. 4 shows the geometry used in the signal model. The ground range from the nadir $(x=0)$ to the swath center is denoted by $x_{c}$, and the whole swath width is $2 x_{o}$. The main lobe width of the spatial filter is denoted by $\Delta \theta_{r x}$, and the boresight angle of the $l$ th spatial filter beam is indicated by the offset $\theta_{l}$ from the incident angle $\theta_{i n c}$. First, only the main lobe signal $r_{l}(t)$ is considered

$r_{l}(t)=\sum_{j=1}^{M_{T}} \int_{x_{n}}^{x_{f}} C_{2 w a y}(\theta) \cdot a_{j}(x) \cdot s_{j}\left(t-\frac{R_{t x, j}+R_{r x}}{c_{o}}\right) \mathrm{d} x$

where $C_{2 w a y}(\theta)$ is the two-way antenna pattern for the elevation angle $\theta, M_{T}$ is the number of transmit antennas, $a_{j}(x)$ denotes the complex scattering coefficient for the OFDM chirp waveform $s_{j}(t)$, and $x_{n}$ and $x_{f}$ denote the nearest and the furthest ground range covered by the main lobe of a spatial filter at the height of $h_{o}$. The subswath width is then given by $x_{f}-x_{n} . R_{t x, j}$ and $R_{r x}$ are the ranges from the target to the phase center of the $j$ th transmit antenna and the receive array, respectively.

In fact, a received signal by a single spatial filter is a superposition of the main lobe and the sidelobe signals, i.e., the signals from a desired subswath and the signals from adjacent other subswaths, respectively. Therefore, it is required that the spatial filtering sufficiently suppresses the sidelobe signals. Put simply, the spatial filtered signal is desired to be equal to the main lobe signal. The performance of the spatial filtering plays a critical role in the demodulation. A further consideration is made to the main lobe width since it determines the received signal length. The main lobe width $\Delta \theta_{r x}$ is defined as $\Delta \theta_{r x}=$ $\theta\left(x_{f}\right)-\theta\left(x_{n}\right)$, and the subswath width $S_{s u b}$ is given by [2]

$$
S_{\text {sub }}=x_{f}-x_{n}=\Delta \theta_{r x} \cdot \frac{h_{o}}{\cos ^{2}\left(\theta_{\text {inc }}-\theta_{l}\right)} \text {. }
$$

The spatial filtering leads to a selection of the subswath, which corresponds to a specific range of delay times. The delay length $\Delta \tau$ of a single subswath is proportional to the main lobe width. Using (9), the relation between $\Delta \tau$ and $\Delta \theta_{r x}$ is formulated as follows:

$$
\begin{aligned}
\Delta \tau & =2 \cdot S_{\text {sub }} \cdot \frac{\sin \left(\theta_{i n c}-\theta_{l}\right)}{c_{o}} \\
& =2 \cdot \Delta \theta_{r x} \cdot \frac{h_{o} \cdot \tan \left(\theta_{i n c}-\theta_{l}\right)}{c_{o} \cdot \cos \left(\theta_{i n c}-\theta_{l}\right)} .
\end{aligned}
$$

In spite of the spatial filtering, the signal length within the main lobe is still longer than the required demodulator (DFT) length $2 N$. The following section presents a processing step to convert the spatial filter signal to fit the length $2 N$.

\section{B. Circular-Shift Addition}

The approach presented in this section is to exploit the periodicity of the discrete Fourier transform, which implies that a finite segment of DFT is a single period of an infinitely extended periodic signal [8]. In the case of periodic signals, the time shift is represented by the phase rotation. If the time shift reaches the signal period length, the phase rotation via the shift becomes $2 \pi$, and the original and shifted signals are no longer distinguishable. Therefore, on the premise that the maximum delay length does not exceed a signal period, any delay of the periodic signal can be represented within the single period. Since the OFDM chirp pulse is a combination of two successive chirps with the length of $T_{p}$ (see Fig. 2), the echo delay of $T_{p}$ results in the $2 \pi$ phase rotation. Therefore, the spatial filter signal $r_{l}(t)$ must satisfy the following strict condition.

Lemma 1: The maximum delay length $\Delta \tau$ of a subswath must be shorter than $T_{p}\left(\Delta \tau<T_{p}\right)$.

Assuming the maximum delay index of $K$ within a subswath $(K<N)$, each delay is described as $(n-k) \cdot T_{s}$, where $k=$ $0,1,2, \ldots, K-1$. Using these discrete time indices $n$ and $k$, the received signal is given in a discrete signal form of

$$
\begin{array}{r}
r_{l}[n]=\left(\sum_{j=1}^{M_{T}} \sum_{k=0}^{K-1} C_{2 w a y}[k] \cdot a_{j}[k] \cdot s_{j}[n-k]\right) \\
\cdot \operatorname{rect}\left[\frac{n}{2 N+K}\right]
\end{array}
$$

where $n=0,1,2, \ldots, 2 N+K-1$. Note that the echo signal length within the subswath is equal to $(2 N+K) \cdot T_{s}$ which is the sum of the OFDM pulse length $\left(2 T_{p}=2 N T_{s}\right)$ and the delay length $\left(K T_{s}\right)$. If the first $2 N$ samples are directly taken by a DFT window, the $2 N$ data samples contain only one entire waveform, and the rest are truncated by the DFT window. In this case, even though the IDFT/DFT is synchronized, its result will be spoiled. To resolve this problem, we introduce an additional 


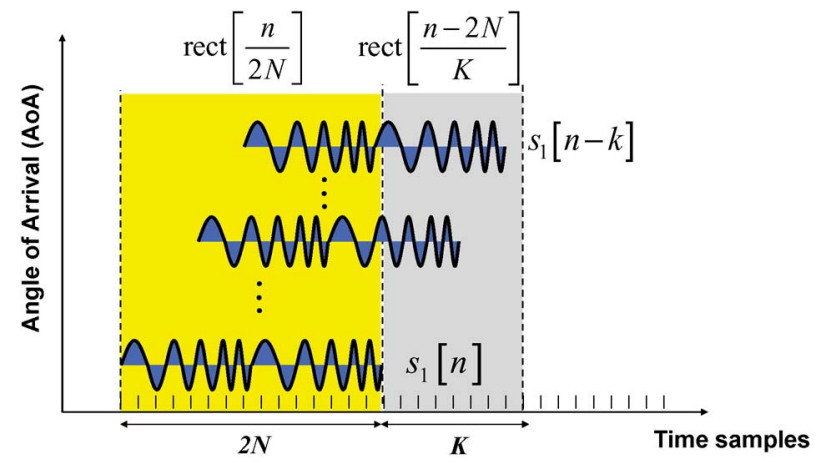

(a)

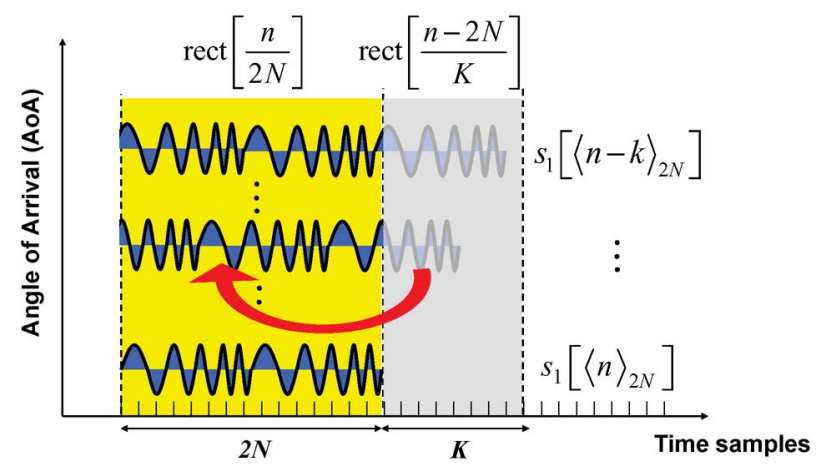

(b)

Fig. 5. Received signals from the subswath with the length (a) before and (b) after the circular-shift addition.

step, called circular-shift addition. This process brings the same effect with the CP into the received signal. The signals before and after the circular-shift addition are shown in Fig. 5(a) and (b), respectively. Using two successive time windows, each with lengths of $2 N$ and $K$, respectively, the signal is divided into two parts. The second term on the right-hand side is circularly shifted and added to the first term in order to make the main lobe signal periodic at each $2 N$ sample. The circular-shift addition process can be described by the modulo operation

$$
r_{l}[n]=\sum_{j=1}^{M_{T}} \sum_{k=1}^{K} C_{2 w a y}[k] \cdot a_{j}[k] \cdot s_{j}\left[\langle n-k\rangle_{2 N}\right]
$$

where $n=0,1,2, \ldots, 2 N-1$ and $\langle\cdot\rangle_{2 N}$ denotes the arithmetic modulo $2 N$. Therefore, the circular-shift addition reduces the signal length from $2 N+K$ to $2 N$ [see Fig. 5(b)]. Following this, the first $2 N$ samples contain all delayed waveforms so that no truncation of the signal occurs. This procedure can be replaced by the residual video phase correction technique [9].

\section{Demodulation With 2N-DFT}

The traditional demodulation by DFT with the length $2 N$ is now applicable to the OFDM chirp signals. Since the transmitted signal $s_{j}[n]$ is the inverse Fourier transform of its spectrum $S_{j}[p]$, the Fourier transform of (12) is described as

$$
\begin{aligned}
& \mathcal{F}\left\{r_{l}[n]\right\}=\frac{1}{2 N} \sum_{n=0}^{2 N-1} \sum_{j=1}^{M_{T}} \sum_{k=0}^{K-1} C_{2 w a y}[k] \cdot a_{j}[k] \\
& \cdot \sum_{p=0}^{2 N-1} S_{j}[p] \cdot \exp \left(j \frac{2 \pi}{2 N}(n-k) p\right) \cdot \exp \left(-j \frac{2 \pi}{2 N} n p\right) .
\end{aligned}
$$

Rearranging (13) with respect to indices, the following formula is obtained:

$$
\mathcal{F}\left\{r_{l}[n]\right\}=\sum_{j=1}^{M_{T}} \sum_{k=0}^{K-1} C_{2 w a y}[k] \cdot a_{j}[k] \cdot \exp \left(-j \frac{2 \pi}{2 N} k p\right) \cdot S_{j}[p]
$$

where $S_{j}[p]$ for $j=1,2$ is derived in (2) and (3). Finally, the demodulated signal is given by

$$
\mathcal{F}\left\{r_{l}[n]\right\}=H_{1} \cdot S_{1}[p]+H_{2} \cdot S_{2}[p]
$$

where $H_{1}$ and $H_{2}$ denote the channel transfer functions delivered by the OFDM waveforms $s_{1}$ and $s_{2}$, respectively. The demodulated signal is indeed separable with respect to each waveform by the polyphase decomposition, which divides the demodulated signal spectrum into odd and even components. Hence, each spectrum length is reduced by $N$. This reduction never leads to any loss of information since the waveforms $s_{1}$ and $s_{2}$ are actually carried by $N$ subcarriers, and the other $N$ subcarrier frequencies are redundant zeros, so as to apply $2 N$-point DFT in demodulation. The channel transfer functions $H_{1}$ and $H_{2}$ can be recovered by the conventional matched filtering for each waveform

$$
H_{1}=\sum_{k=0}^{K-1} C_{2 w a y}[k] \cdot a_{1}[k] \cdot \exp \left(-j \frac{2 \pi}{N} k \frac{p}{2}\right) \cdot\left|S_{1}\right|^{2} .
$$

In the same manner, the channel transfer function $H_{2}$ is recovered

$$
\begin{array}{r}
H_{2}=\sum_{k=0}^{K-1} C_{2 w a y}[k] \cdot a_{2}[k] \cdot \exp \left(-j \frac{2 \pi}{N} k \frac{p}{2}\right) \\
\cdot \exp \left(-j \frac{\pi}{N} k\right) \cdot\left|S_{2}\right|^{2}
\end{array}
$$

whereby the second exponential term accounts for the linear phase ramp depending on the target delay, which is caused by the subcarrier spacing $\Delta f$. Therefore, there is a linear phase difference between two images reconstructed by each OFDM chirp waveform, which can be easily compensated in the time domain.

\section{VERIFICATION}

The OFDM chirp waveform scheme is verified by simulations in this section. The simulation is carried out under the following assumptions: use of the optimum spatial filter and the simple delay channel (point scatterers). The optimum spatial filter means that the antenna pattern is ideally covering the angular range of interest and suppressing the sidelobe signals. The channel is assumed only to cause delay and to include the thermal noise. The simulation schematic is shown in Fig. 6. The input sequence length $N$ is equal to 1024 so that a total of 2048 subcarriers are used for the modulation and demodulation within the bandwidth of $100 \mathrm{MHz}$. The modulated OFDM chirp length $2 T_{p}$ is $17.067 \mu$ s. Hence, the maximum delay of each channel never exceeds a $T_{p}$ of $8.53 \mu$ s. These OFDM waveforms travel through two different channels, $h_{1}$ and $h_{2}$, and return to the receiver. Both of them are processed by a single demodulator (receiver). 


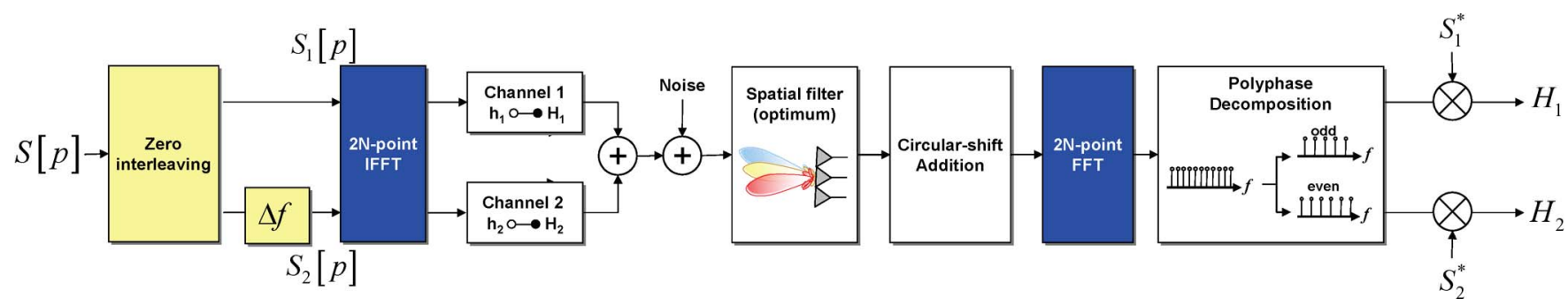

Fig. 6. Simulation schematic for verification: Two independent channels and a single demodulator with DBF.

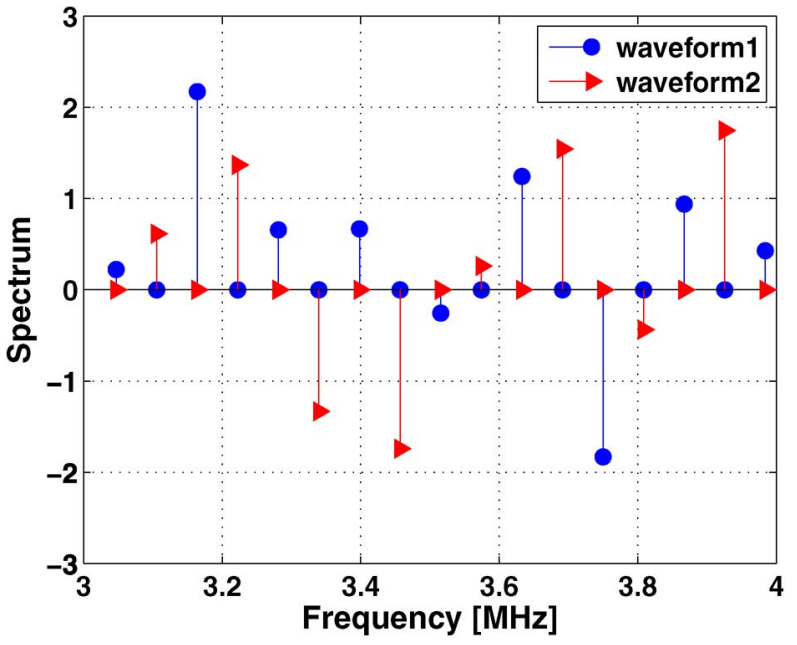

Fig. 7. OFDM demodulated discrete spectra of both waveforms within 3 and $4 \mathrm{MHz}$. The circular and triangular symbols indicate the spectral components (subcarriers) of $H_{1} S_{1}$ and $H_{2} S_{2}$, respectively. $\Delta f=58.59 \mathrm{kHz}$.
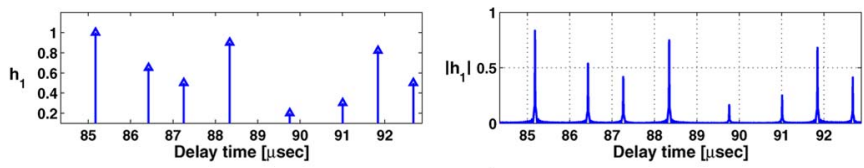

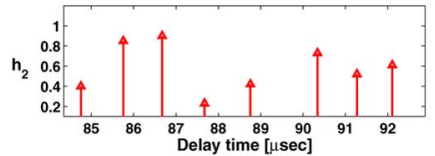

(a)

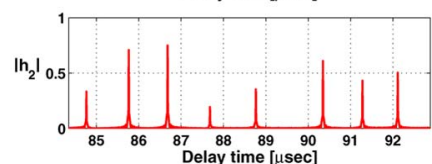

(b)
Fig. 8. Impulse response functions (range profiles) of channel $h_{1}$ and $h_{2}$ : (a) Original channel impulse response functions and (b) the reconstructed impulse response functions from the OFDM chirp signals.

Fig. 7 presents the spectra of demodulated signals within a $1-\mathrm{MHz}$ range from 3 to $4 \mathrm{MHz}$ for clarity. The circular symbols denote the spectral components of $H_{1} S_{1}$, and the triangular symbols indicate them for $\mathrm{H}_{2} \mathrm{~S}_{2}$. It is clearly observed that these two spectral components are out of joint, i.e., orthogonal.

Fig. 8(a) shows the original channel impulse response functions $h_{1}$ and $h_{2}$, defined for this simulation. These channels contain several impulses, indicating the delay and scattering coefficient of each target. The reconstructed channel impulse responses are plotted in Fig. 8(b). Apart from a slight change of the peak magnitude due to the Fourier transform of the band-limited signal, both range profiles recovered by the single demodulator and independent matched filters correspond to the original channel impulse response functions.

\section{Conclusion}

In this letter, we proposed a novel waveform scheme, based on the principle of OFDM technique combined with conventional chirp waveforms. Regarding a typical spaceborne SAR scenario, this letter dealt with the demodulation scheme combined with DBF and verified it by simulation. The proposed OFDM chirp waveforms preserve their orthogonality, as long as they are processed by the presented demodulation scheme. As a consequence, we intend to emphasize the following features: First, the OFDM chirp waveform has the same characteristics of the original input chirp signal. This aspect will be of a special interest for the implementation of the MIMO SAR system with the modern hardware and digital synthesis technologies. Second, the novel waveform scheme has a high adaptability, which implies that the novel waveform scheme can be easily combined with other waveform schemes, such as the space-frequency coding [10] or the multidimensional encoding technique [11]. Finally, owing to the common spectral band, the scheme shows high potential to use multiple SAR images produced using OFDM chirps for polarimetric and interferometric applications. Future research will include the performance for the proposed approach for distributed targets.

\section{REFERENCES}

[1] J. Kim, "Multiple-input multiple-output synthetic aperture radar for multimodal operation," Ph.D. dissertation, Karlsruhe Inst. Technol. (KIT), Karlsruhe, Germany, 2011.

[2] J. C. Curlander and R. N. McDonough, Synthetic Aperture Radar Systems and Signal Processing. New York: Wiley, 1991.

[3] R. W. Chang, "Orthogonal Frequency Division Multiplexing," U.S. Patent 3488 445, Jan. 1970.

[4] A. Paulraj, R. Nabar, and D. Gore, Introduction to Space-Time Wireless Communications. Cambridge, U.K.: Cambridge Univ. Press, 2003.

[5] D. Garmatyuk, "Adaptive multicarrier OFDM SAR signal processing," IEEE Trans. Geosci. Remote Sens., vol. 49, no. 10, pp. 3780-3790, Oct. 2011.

[6] W. Wang, "Space-time coding MIMO-OFDM SAR for high-resolution imaging," IEEE Trans. Geosci. Remote Sens., vol. 49, no. 8, pp. 30943104, Aug. 2011.

[7] N. Levanon and E. Mozeson, Radar Signals. Chichester, U.K.: Wiley, 2004.

[8] A. Oppenheim, A. Willsky, and S. Nawab, Signals and Systems, 2nd ed. Upper Saddle River, NJ: Prentice-Hall, 1997.

[9] J. Mittermayer, A. Moreira, and O. Loffeld, "Spotlight SAR data processing using the frequency scaling algorithm," IEEE Trans. Geosci. Remote Sens., vol. 37, no. 5, pp. 2198-2214, Sep. 1999.

[10] J. Kim and W. Wiesbeck, "Investigation of a new multifunctional high performance SAR system concept exploiting MIMO technology," in Proc. Int. Geosci. Remote Sens. Symp., Boston, MA, Jul. 2008, vol. 2, pp. II-221-II-224.

[11] G. Krieger, N. Gebert, and A. Moreira, "Multidimensional waveform encoding: A new digital beamforming technique for synthetic aperture radar remote sensing," IEEE Trans. Geosci. Remote Sens., vol. 46, no. 1, pp. 31-46, Jan. 2008. 